\title{
Correction to: Microstates, Entropy and Quanta
}

\section{Correction to: \\ D. Koks, Microstates, Entropy and Quanta, https://doi.org/10.1007/978-3-030-02429-1}

In the original version of the book, the following belated corrections have been incorporated: In Chapters 1 and 2, typographical errors in both text and mathematical equations have been corrected. The book and the chapters have been updated with the changes. 